\title{
Swabbing: touchscreen-based input technique for people with hand tremor
}

\author{
Alexander Mertens ${ }^{\mathrm{a},{ }^{*}}$, Jan Hurtmanns ${ }^{\mathrm{a}}$, Chatchavan Wacharamanotham ${ }^{\mathrm{b}}$, Martin Kronenbürger ${ }^{\mathrm{c}}$, \\ Jan Borchers ${ }^{\mathrm{b}}$, and Christopher M. Schlick ${ }^{\mathrm{a}}$ \\ ${ }^{a}$ Institute of Industrial Engineering and Ergonomics, RWTH Aachen University, \\ Bergdriesch 27, 52062 Aachen, Germany \\ ${ }^{\mathrm{b}}$ Media Computing Group, RWTH Aachen University, Ahornstraße 55, 52074 Aachen, Germany \\ ${ }^{\mathrm{c}}$ Department of Neurology, Medical Faculty, RWTH Aachen, Pauwelsstraße 30,52074 Aachen, Germany
}

\begin{abstract}
Interacting with touchscreen computer systems is a challenging task for elderly tremor patients, because of a lower input accuracy caused by their tremor symptoms. Previous observations by Mertens et al. suggested that continuous movements are able to lower these symptoms. In this paper Swabbing, a input method for touchscreen systems based on such motions, is presented. A user study will show that Swabbing is able to reduce the error rate while proving equal user satisfaction compared to standard input techniques. Furthermore reasons for these benefits will be argued and explained with the use of accuracy measures by MacKenzie et al. In the end possible future enhancements of Swabbing will be discussed.
\end{abstract}

Keywords: HCI, touchscreen, older adults, Swabbing, tremor

\section{Introduction}

In the past years the Demographic Change has led to fundamental changes in the population of the industrialized countries. Overall the ratio of elderly people increased strongly and will most probably continue to do so in the next years [1].

This change will also raise the number of tremor patients, based on the fact that the ratio of people with tremor increases with age [11].

Another currently observable trend is the increasing use of computers in general [18] and of touchscreen system in particular [4]. Especially motionimpaired users can benefit from this growth since computer technology offer them great prospects when it comes to educational success, employment opportunities and social communication [3]. Unfortunately these people reach significant lower levels of input accuracy compared to able-bodied users when interacting with computer systems [15].

These two trends lead to the conclusion that in all probability older people will use computer systems more often in the future.
Additionally previous research had shown that the use of direct input devices like touchscreens offers lots of advantages when used for assistive technology. Rogers et al. showed that touchscreens technology can be used with minimal training, that it reduces the cognitive load for the user in general, as well as the physical workload for elderly, and has also a high user acceptance [17].

The presented facts show that a technique that makes touchscreens easier accessible for elderly tremor patients is needed.

\section{Related Work}

Older aged tremor patients have a lower input efficiency when using a computer compared to ablebodied users. An investigation of the specific problems of tremor users with common input devices (mouse, joystick) was done in different related works. These studies resulted in several interesting details.

Keates et al. analyzed the movements of motionimpaired and able-bodied users during point-and-

*Corresponding author. E-Mail: a.mertens@iaw.rwth-aachen.de 
click tasks [12]. They used an extended version of MacKenzie's measurement model for cursor movement [14]. The evaluations of these tests showed interestingly that motion impaired users have no significant higher movement variability (MV). That means that their motion on the way to a target do not deviate significantly more than for able bodied users. However, they have more target re-entries (TRE), which means that they need many attempts to get over a target.

Findlater et al. found out that clicking on a target is especially complex for motion-impaired users since it requires a high level of precision control [5]. This observation was also made by Hwang et al. who noticed that motion-impaired users often pass over a target or slip off it during the click [10].

Keates and Trewin got similar results from their studies with elderly Parkinson patients. Their experiments showed that their users faced the problem of staying over a target long enough to click and that many slip off errors occurred when they finally managed to do so. In addition long pauses were made while moving around or through the target [13].

Overall it should be stated that people with motion impairments can easily move their finger towards a target but it is demanding for them to maintain their finger accurately over it.

Although these observations were not all made with tremor patients the results were assumed to be valid for them too. However it is suspected that the movement variability is higher for tremor patients, especially for those having a resting tremor.

In the past many research had been done on the field of HCI to allow motion impaired people to operate with computer systems properly. A general approach to address their specific problems is the use of additional hardware.

Camera based systems like the Camera Mouse, allows to interact with computers by tracking various parts of the body like the nose or chin and map these movements to the corresponding cursor position [2]. Like all tracking based solutions, the Camera Mouse is suitable to only a limited extent for tremor patients since their symptoms will cause their whole body to tremble even if only limited parts of the body - like their hands - are directly affected from tremor.

The same problem applies for the use of voice recognition in general since the mouse and tongue of tremor patients are also likely to shake because of their symptoms which will complicate the recognition.

Another way to ease the computer interaction for motion-impaired users is the use of software-based solutions.

Gajos et al. proposed various methods which generate personalized interfaces for motion impaired users automatically [6-9]. In general a mixture of collecting the preferences for user interfaces a priori and allow them to choose between different interface fragments with the same functionality a posteriori is used. Even though these results show an advantage for many different motion-impaired users, the characteristic symptoms of tremor patients are not taken into consideration for this work. By rearranging interface parts and using alternative UI elements the main problem of users with tremor, the performing of an exact click on a target, is not solved.

This problem can be generally addressed by increasing the target size. Thinking of modern graphical user interfaces (GUIs) this option can only be used to a certain point. First the size of screens serves as a physical limitation for enhancing buttons. When thinking of smaller devices this space will become even smaller in the future. In addition it must be considered that bigger UI elements result in less space for content displaying.

Although much effort was put in developing new systems that help motion-impaired users interacting with computer systems more efficiently, it must be stated that most of the systems do not consider the specific symptoms tremor patients have to deal with. Furthermore using direct input methods on touchscreens for individuals with tremor is only roughly investigated and currently there is no solution to that specific problem.

\section{Swabbing}

The main idea behind Swabbing is to use a motion towards a target for the actual target selection to circumvent the specific problems of tremor users while getting and staying over a target before tapping on it.

\subsection{GUI design}

To allow such movements the targets for the user study GUI were all evenly distributed arranged along the screen border as can be seen in Figure 1 . 
Since the users are allowed to start their motion everywhere on the screen corridor lines were added between the targets, to serve as a visual hint in which direction the swabbing movement should be made to activate a target. Furthermore an area in the middle was left free to give the user a possible starting area.

The targets itself were depicted by arrows which were found beneficial for the understanding of Swabbing in previous studies.

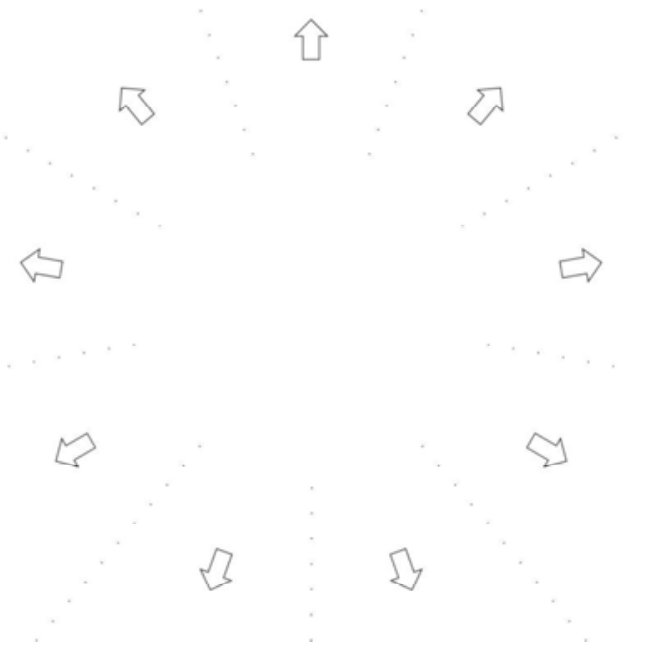

Fig. 1: Swabbing GUI design

\subsection{Algorithm}

A Swabbing motion can be divided into three phases. The detection of a motion begins when the user presses his finger on the touchscreen (TouchDown).

After that all further touchpoints are cached, as long as a permanent contact with the surface exists. This continuous contact with the touchscreen is recognized as an input action, when a specific Euclidean distance (threshold) between the first touchpoint and the current one is reached. For the user studies a value of $250 \mathrm{px}$ was used, which is approximately half of the mean distance between the screen center and border. When this happens all collected touchpoints are used to determine a linear function via the method of ordinary least squares (OLS).

In the next step this line is converted to a vector by looking at the first and last touchpoint coordi- nates. Subsequently this vector is used to identify the point on the screen border that was targeted by the user.

With the help of this information the intended target can be identified later. If the contact is lost (TouchUp) before the threshold is reached, a timer of $200 \mathrm{~ms}$ starts. If the finger returns to the screen (TouchDown) during this period, the motion continues without any effect.

Otherwise the motion is aborted and the touchpoint cache is cleared. This mechanism was included to prevent that an involuntary lift of the finger that is caused by the tremor results in a direct abort of the motion.

\section{Study Setup}

The user test compared the target selection accuracy between Swabbing and the current standard interaction technique, Tapping. Therefore three different GUI's, Swabbing, Radial and Grid, were used to perform a selection test. Two different target arrangements, a radial and a grid layout, were used for Tapping to test whether or not the layout of targets influences the accuracy.

In addition a two-part user interview was conducted. The first part was interviewed before the aforementioned user test. It was asked for requested general user information whereas the second part, a PSSUQ, was used to gain feedback after the test.

The study was performed in a quiet room with sufficient light. The testing was done on a HP TouchSmart tm2-1090eg in tablet mode that was fixed into a customized stand that allows inclining the touchscreen from the desk surface accurately. By doing so it could be ensured, that the degree between screen and table is $20^{\circ}-$ a degree that was discovered to be optimal for using touchscreens [16].

After an initial greeting the participants were asked to select on-screen targets in different target resolutions 9, 16, 25 and in three conditions: Tapping+Grid layout, Tapping+Radial layout, Swabbing+Radial layout.

In each condition, the participant had to select targets until he had done 10 successful selections.

The trials for each method were performed for ascending resolutions. Latin Square was used to counterbalance the order of conditions to prevent learning and boredom effects. 


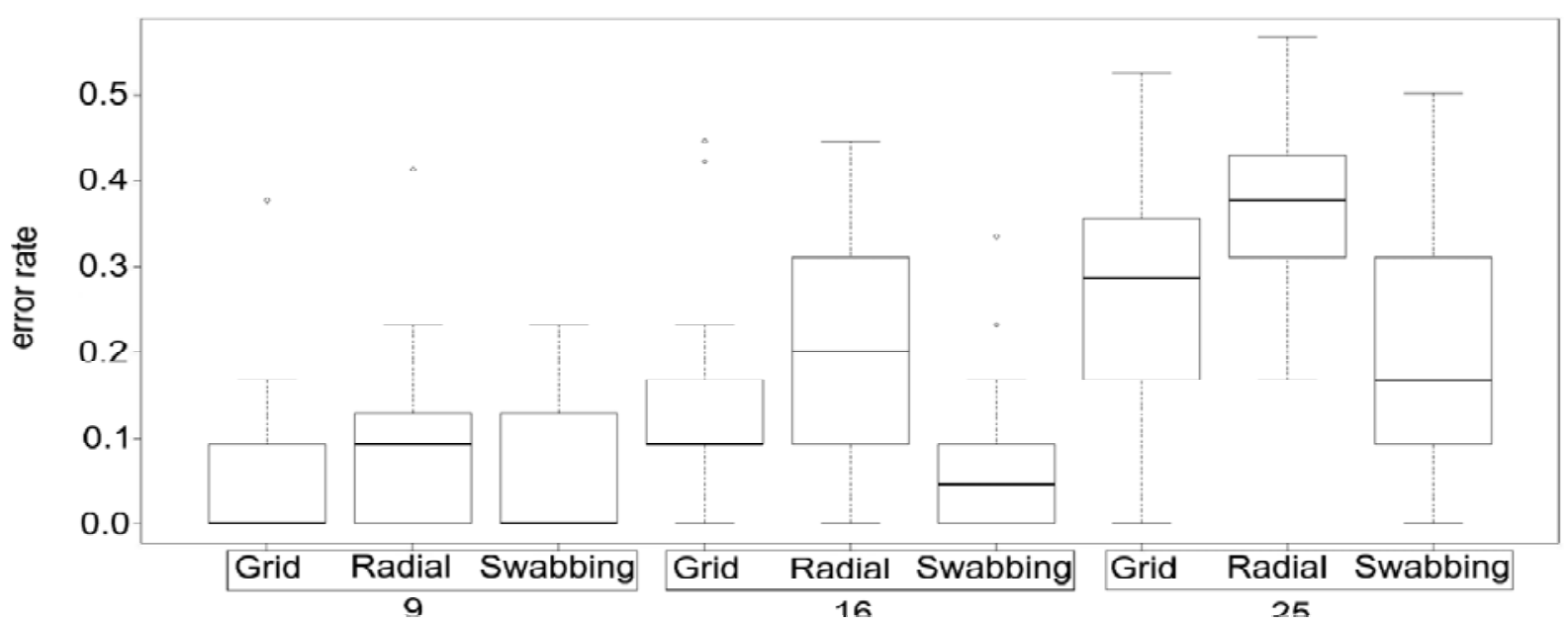

Fig. 2: Error rate overview

The interaction area for all three visualizations was $800 \times 800$ pixels in the center of the screen. For Grid the whole interaction area was filled with buttons that were arranged in a grid pattern. In Radial the buttons were arranged radial comparable to Swabbing. To activate a button in these Tapping visualizations, the user had to press on it with his finger.

For all visualizations, targets that should be selected were highlighted blue. The targets flashed red upon wrong selections and accordingly green after correct ones.

Next to the users dominant hand side of the interaction area, a crosshairs in the middle of a blackened area designated the parking position where the user's

finger had to return before he could continue with the next trial. This was done to assure that each movement began at the same point for every selection.

During the study the number of errors as well as all touchpoint coordinates were collected to use them for further accuracy measurements.

Sixteen participants with tremor attended at this user study. Table 1 summarizes the general information of the participants that were taken from the first part of the questionnaire.

\section{Results and Discussion}

\subsection{Study results}

The trial data analysis began by using two-way repeated measures ANOVA models with significance level $\alpha=0.05$.

Table 1: User study participants' summary

$\begin{array}{lllll}\text { User\# } & \text { Age } & \text { Gender } & \text { Tremor type } & \text { Tremor amplitude } \\ 1 & 74 & \mathrm{~m} & \text { Intention } & \text { severe }>2 \mathrm{~cm} \\ 2 & 77 & \mathrm{~m} & \text { Rest } & \text { slight }<0,5 \mathrm{~cm} \\ 3 & 79 & \mathrm{~m} & \text { Intention } & \text { marked } 1-2 \mathrm{~cm} \\ 4 & 62 & \mathrm{~m} & \text { Intention } & \text { slight }<0,5 \mathrm{~cm} \\ 5 & 74 & \mathrm{~m} & \text { Intention } & \text { severe }>2 \mathrm{~cm} \\ 6 & 58 & \mathrm{f} & \text { Intention } & \text { moderate } 0,5-1 \mathrm{~cm} \\ 7 & 82 & \mathrm{f} & \text { Intention } & \text { moderate } 0,5-1 \mathrm{~cm} \\ 8 & 76 & \mathrm{~m} & \text { Intention } & \text { marked } 1-2 \mathrm{~cm} \\ 9 & 73 & \mathrm{~m} & \text { Rest } & \text { slight }<0,5 \mathrm{~cm} \\ 10 & 71 & \mathrm{~m} & \text { Rest } & \text { marked } 1-2 \mathrm{~cm} \\ 11 & 75 & \mathrm{f} & \text { Intention } & \text { moderate } 0,5-1 \mathrm{~cm} \\ 12 & 87 & \mathrm{~m} & \text { Intention } & \text { severe }>2 \mathrm{~cm} \\ 13 & 70 & \mathrm{~m} & \text { Intention } & \text { severe }>2 \mathrm{~cm} \\ 14 & 82 & \mathrm{~m} & \text { Intention } & \text { marked } 1-2 \mathrm{~cm} \\ 15 & 68 & \mathrm{f} & \text { Intention } & \text { severe }>2 \mathrm{~cm} \\ 16 & 73 & \mathrm{f} & \text { Intention } & \text { moderate } 0,5-1 \mathrm{~cm}\end{array}$

To make sure that a comparison of the different layouts was possible it was checked whether the results contained interaction between the interaction techniques and the different resolutions. 

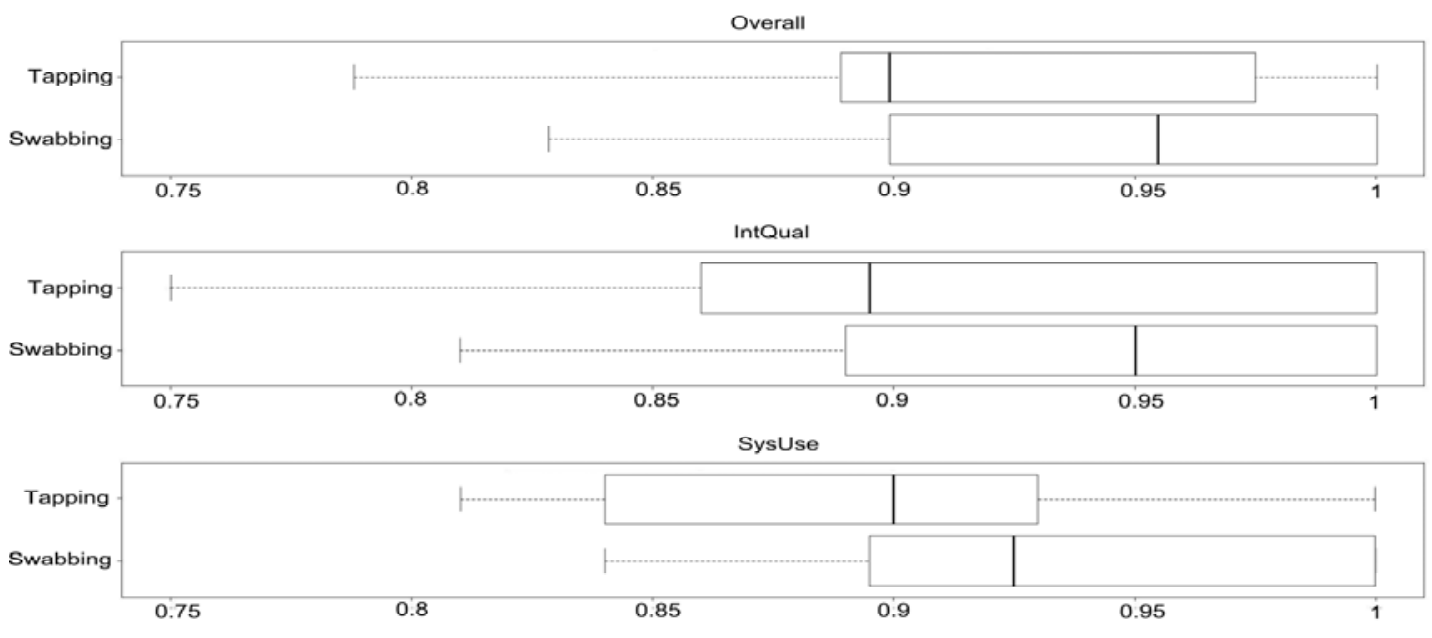

Fig. 3: PSSUQ data overview

The difference between the used interaction techniques were found significant for Swabbing and Radial $(\mathrm{F}=23.316 ; \mathrm{p}<0.001)$ as well as for Swabbing and Grid $(\mathrm{F}=4.2925 ; \mathrm{p}<0.05)$.

Figure 2 illustrates that the error rate of Swabbing is considerable less for 16 and 25 targets. Pairwise ttest with Bonferroni corrections confirmed these results $(\mathrm{p}<0.05$ in every case $)$.

The logged touchpoints were used to calculate the MV (movement variability) of each participant for each resolution. For each target selection the collected touchpoints were used to determine to which extend all points lie along a perfect line to the target.

In our case the computated linear regression lines were used as the reference lines.

$$
M V=\sqrt{\frac{\sum\left(y_{i}-\bar{y}\right)^{2}}{n-1}}
$$

The resulting values show an average MV of 5.5819 with a standard deviation of 4.107.

The data from the PSSUQ were used to analyze the user satisfaction with Tapping and Swabbing. Figure 3 shows the results of the questionnaire for the two techniques.

Although Swabbing has higher ratings in all categories only the results for the system satisfaction (chi-squared $=5.4444$, df $=1, \mathrm{p}<0.05$ ) and usability (chi-squared $=4.4545, \mathrm{df}=1, \mathrm{p}<0.05$ ) could be proven significant by applying Friedman tests.

\subsection{Discussion}

The results of the user study show that Swabbing is able to reduce the error rate compared to Tapping significantly. This effect seems to increase with a higher number of symbols, which makes it especially interesting for scenarios like text input.

The calculation of the movement variability seems to indicate that the basic idea of Swabbing works in practice. Compared to the values of able bodied users for normal point and click operations on a touchscreen (average $=11.7$, standard deviation = 2.4) measured by MacKenzie et al. [14], Swabbing reaches a surprisingly even level. This is most probably based on the fact that continuous motions are considerably easier to perform for tremor patients. Furthermore Swabbing seems to be able to make use of this effect in the praxis.

When thinking of a future use of Swabbing in a real world scenario it is of intense importance that the technique is accepted by the peer group. The results from the PSSUQ suggest that acceptance for Swabbing is at least as high as for Tapping although it was a complete new technique to the test persons.

The fact that all patients became quickly familiar with the technique during the user study supports this impression. 


\section{Summary and Future Work}

\subsection{Summary}

In this paper Swabbing, a new input method that addresses the specific problems of elderly tremor patients when interacting with touchscreens, was presented. Swabbing should fill a gap in assistive technology and make touchscreen technology accessible for older people that are affected by tremor.

In contrast to the standard touchscreen input method, Tapping, Swabbing uses a continuous movement towards the target for selection. Therefore Swabbing reduces the strong tremor symptoms that occur when tremor patients try to reach and stay over a target. A finger movement trajectory, which determines a target, is calculated using linear regression. This reduces noisy movements caused by tremor.

A user study presented in this work compared Swabbing with Tapping. It is shown that Swabbing has a significantly lower error rate for 16 , and 25 symbols. Furthermore the user questionnaire revealed that satisfaction with Swabbing is higher than with Tapping.

The limitation of the studies was a small number of participants. It was not possible to narrow the participants to one type of tremor because of the lack of users who matches the conditions. Although this does not diminish the significance of the results, the readers should keep this in mind. Overall Swabbing has proven to be a promising new input method that has significant advantages over Tapping for elderly tremor patients interacting with touchscreens.

\subsection{Future Work}

Although it has been shown that Swabbing is a promising input method, there are various possible refinements of the input recognition which can be done in the future.

This may be done by using alternative smooth algorithms that may increase the accuracy of the input.

For example the algorithm may be personalized to accommodate the individual tremor variations. The input threshold may also be personalized.

Later, more complex algorithm increments like the use of heuristics will be a possible advancement. Therefore probability distributions of the users language can be used to determine how likely a charac- ter is to be the next one. The angles for each character section may then be adjusted accordingly.

Furthermore Swabbing should be implemented as a word processing technique and compared to Tapping in a real world scenario.

\section{Acknowledgment}

This research is funded by the German Federal Ministry of Education and Research BMBF (01FG10004) and the body responsible for the project is DLR.

\section{References}

[1] G.F. Anderson and P.S. Hussey, Population aging: a comparison among industrialized countries, Health Affairs 19, 2000, 191-203

[2] M. Betke, Camera-based interfaces and assistive software for people with severe motion impairments, Artificial Intelligence Techniques for Ambient Intelligence, 2008

[3] C. Brown, Assistive technology computers and persons with disabilities, Communications of the ACM 35, 1992, 36-45

[4] Roberta Cozza and CK Lu, Forecast: Touchscreen mobile devices 2006-2013, 2011

[5] L. Findlater, A. Jansen, K. Shinohara, M. Dixon, P. Kamb, J. Rakita, and J. O. Wobbrock, Enhanced area cursors, UIST 2010, New York, 2010, page 153

[6] K. Gajos and D. S. Weld, SUPPLE, IUI 2004, Funchal, 2004 , page 93

[7] K. Z. Gajos and D. S. Weld, Preference elicitation for interface optimization, UIST 2005, Seattle, 2005, page 173

[8] K. Z. Gajos, J. O. Wobbrock, and D. S. Weld, Automatically generating user interfaces adapted to users' motor and vision capabilities, UIST 2007, Newport, 2007, page 231

[9] K. Z. Gajos, J. O. Wobbrock, and D. S. Weld, Improving the performance of motor-impaired users with automaticallygenerated, ability-based interfaces, CHI 2008, Florence, 2008, page 1257

[10]F. Hwang, S. Keates, P. Langdon, and J. Clarkson, Mouse movements of motion-impaired users: a submovement analysis, ACM SIGACCESS 2003, 77-78

[11]E. D. Louis, R. Ottman, and W. A. Hause, How common is the most common adult movement disorder? Estimates of the preavalence of essential tremor throughout the world, Movement Disorders 13, 1998, 5-10

[12] Simeon Keates, Faustina Hwang, Patrick Langdon, P. John Clarkson, and Peter Robinson, Cursor measures for motionimpaired computer users, Assets 2002, Edinburgh, page 135

[13]S. Keates and S. Trewin, Effect of age and parkinson's disease on cursor positioning using a mouse, Assets 2005, Baltimore, 2005, page 68

[14]S. MacKenzie, T. Kauppinen, and M. Silfverberg, Accuracy measures for evaluating computer pointing devices, CHI 2001, ACM, New York, 2001, 9-16 
[15]A. Mertens, N. Jochems, C. M. Schlick, D. Duennebacke, and J. H. Dornberg, Design pattern TRABING, EICS 2010, Berlin, 2010, page 267

[16]C. Muller-Tomfelde, A. Wessels, and C. Schremmer, Tilted tabletops: In between horizontal and vertical workspaces, Horizontal Interactive Human Computer Systems, Amsterdam, 2008, 49-56
[17] W. A. Rogers, M. A. O’Brien, and A. C. McLaughlin, Selection and design of input devices for assistive technologies, 9th International Conference on Control, Automation, Robotics and Vision, Singapore, 2006, 1-6

[18] Statistisches Bundesamt, Deutschland - PC und Internet praegen zunehmend Berufs- und Privatleben, 2008 